"NOTICE: this is the author's version of a work that was accepted for publication in Journal of Biomechanics. Changes resulting from the publishing process, such as peer review, editing, corrections, structural formatting, and other quality control mechanisms may not be reflected in this document. Changes may have been made to this work since it was submitted for publication. A definitive version will be subsequently published in Journal of Biomechanics, [VOL.40, pp.2752-2757, (2007)].”

\title{
In vitro confocal micro-PIV measurements of blood flow in a square microchannel: The effect of the haematocrit on instantaneous velocity profiles
}

\author{
R. LIMA ${ }^{1,2}$, S. WADA ${ }^{1}$, M. TAKEDA ${ }^{1,2}$, K. TSUBOTA ${ }^{1}$, T. YAMAGUCHI ${ }^{1}$ \\ ${ }^{1}$ Dept. Bioeng. \& Robotics, Grad. Sch. Eng., Tohoku Univ., 6-6-01 Aoba, 980-8579 Sendai, Japan. \\ ${ }^{2}$ Dept. Mechanical Technology, ESTiG, Bragança Polyt., C. Sta. Apolónia, 5301-857 Bragança, Portugal. \\ ${ }^{3}$ Dept of Mechanical Science and Bioeng., Grad. Sch. Eng Sci., Osaka Univ., Toyonaka, 560-8531 Osaka, Japan \\ ${ }^{4}$ Div. Surgical Oncology, Grad. Sch Medicine, Tohoku Univ., 2-1 Seiryo-machi, Aoba-ku, 980-8575 Sendai, Japan \\ E-mail: rui@pfsl.mech.tohoku.ac.jp
}

\begin{abstract}
A confocal microparticle image velocimetry (micro-PIV) system was used to obtain detailed information on the velocity profiles for the flow of pure water $(P W)$ and in vitro blood (haematocrit up to 17\%) in a 100-mm-square microchannel. All the measurements were made in the middle plane of the microchannel at a constant flow rate and low Reynolds number (Re 1/4 0.025). The averaged ensemble velocity profiles were found to be markedly parabolic for all the working fluids studied. When comparing the instantaneous velocity profiles of the three fluids, our results indicated that the profile shape depended on the haematocrit. Our confocal micro-PIV measurements demonstrate that the root mean square (RMS) values increase with the haematocrit implying that it is important to consider the information provided by the instantaneous velocity fields, even at low Re. The present study also examines the potential effect of the RBCs on the accuracy of the instantaneous velocity measurements.
\end{abstract}

Key words: microcirculation, confocal micro-PIV, Nipkow disk, blood cell suspension, microchannel.

\section{Introduction}

The velocity profiles of blood flow in vivo and in vitro have been measured using several techniques, including double-slit photometry (Gaehtgens et al., 1970; Baker and Wayland, 1974), video microscopy and image analysis (Bugliarello et al., 1963; Tangelder et al., 1986; Parthasarathi et al.,1999), laser-Doppler anemometry (Einav et al., 1975; Born et al., 1978; Cochrane et al., 1981; Uijttewaal et al., 1994, Golster et al., 1999), and particle-measuring methods (Sugii et al., 2002, 2005; Nakano et al., 2003, 2005; Jeong et al., 2006). Nevertheless, no general consensus yet exists concerning the actual velocity profile in microvessels. While some studies have reported parabolic profiles (Baker and Wayland, 1974; Golster et al., 1999; Sugii et al., 2005), others have suggested blunt profiles (Bugliarello et al., 1963; Tangelder et al., 1986; Nakano et al., 2003); still others have reported blunt profiles at extremely low velocities and diameters and parabolic profiles at diameters exceeding $100 \mu \mathrm{m}$ (Gaehtgens et al., 1970; Cochrane et al., 1981). Thus, further research is needed on the influence of flow parameters on the blood flow velocity profiles in microvessels.

Due to its outstanding spatial filtering technique and multiple point light illumination system, confocal micro-PIV (Tanaani et al., 2002; Park et al., 2004, 2006; Kinoshita et al., 2005; Lima et al., 2006) has become accepted as a reliable method for measuring velocity profiles with high spatial resolution. Very recently, we demonstrated the ability of confocal micro-PIV to measure both homogeneous and nonhomogeneous fluids (Lima et al., 2006). The present study compared the instantaneous and 
ensemble velocities profiles of pure water and blood flow in vitro. The velocity profiles of both pure water and in vitro blood with two different haematocrits $(9 \% \mathrm{Hct}$ and $17 \% \mathrm{Hct})$ were acquired in the centre plane of a $100 \mu \mathrm{m}$ square microchannel.

\section{Materials and methods}

\subsection{Working fluids and microchannel}

This study examined three working fluids: pure water (PW) and physiological saline (PS) containing $9 \%$ (9Hct) or $17 \%$ (17Hct) human red blood cells (RBCs). All the fluids were seeded with $0.15 \%$ (by volume) 1- $\mu$ m-diameter red fluorescent solid polymer microspheres (R0100; Duke Scientific, Palo Alto, CA, USA). The blood used was collected from a healthy adult volunteer (aged 32 years old), and ethylenediaminetetraacetic acid (EDTA) was added to prevent coagulation. The RBCs were separated from the bulk blood by centrifugation (1500 rpm for $20 \mathrm{~min}$ ) and the plasma and buffy coat were removed by aspiration (see Figure 1). The washing and centrifuging with PS was repeated twice. The remaining RBCs were then resuspended in PS to make up the required RBC concentration by volume. The haematocrit of the two RBC suspensions used in this study was about 9\% (9Hct) and 17\% (17Hct), respectively. All the blood samples were stored hermetically at $4^{\circ} \mathrm{C}$ until the experiment was performed at room temperature $\left(25-27^{\circ} \mathrm{C}\right)$. All procedures in this experiment were carried out in compliance with the Ethics Committee on Clinical Investigation of Tohoku University.

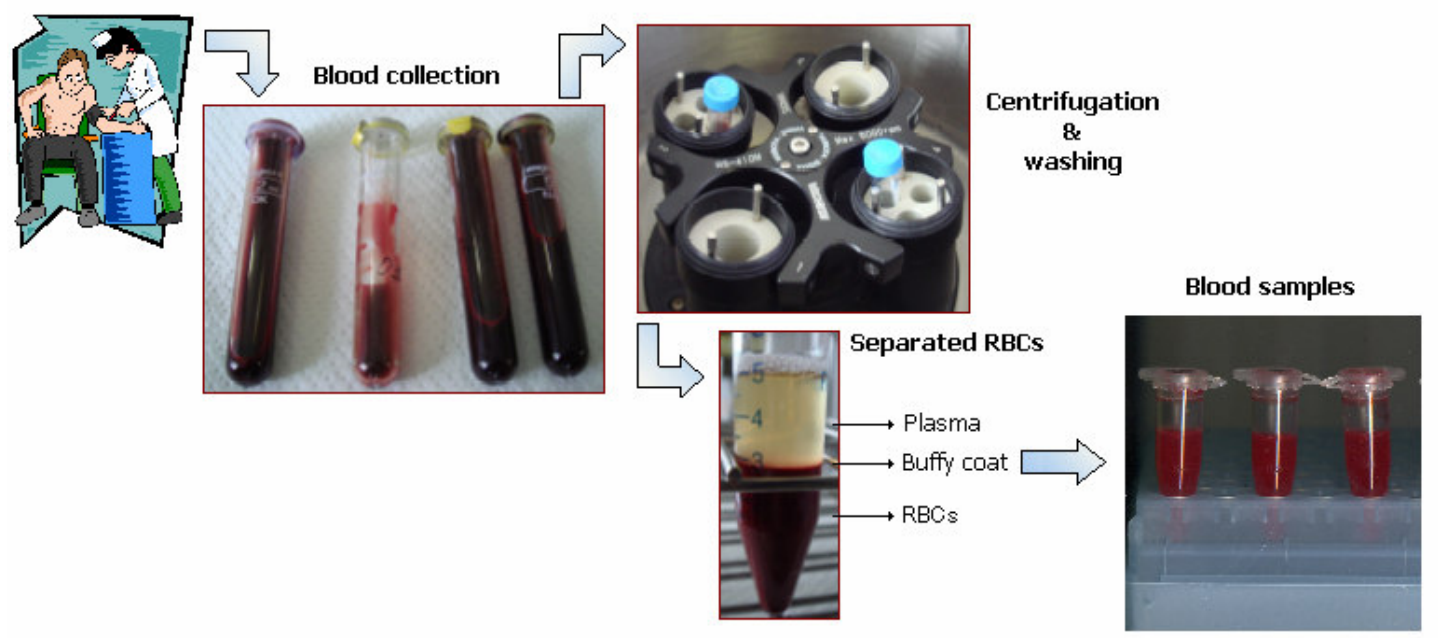

Figure 1 Preparation of the blood samples.

In this study, we used a $100 \mu \mathrm{m}$ square borosilicate glass microchannel fabricated by Vitrocom (Mountain Lakes, NJ, USA), which was mounted on a slide glass immersed in glycerol that had the same refractive index. A square microchannel was selected to minimise possible refraction of the laser beam at the walls of the microchannel. Using a glass tube, the refraction arising from the curved walls would be more pronounced and might degrade the measured velocity fields.

\subsection{Experimental setup}

The confocal micro-PIV system we used consisted of an inverted microscope (IX71; Olympus, Tokyo, Japan) combined with a confocal scanning unit (CSU22; Yokogawa Corp., Tokyo, Japan) and a diodepumped solid-state (DPSS) continuous wave (CW) laser (Laser Quantum, Stockport, UK) with an excitation wavelength of $532 \mathrm{~nm}$. A high-speed camera (Phantom v7.1; Vision Research, Wayne, NJ, USA) was connected to the outlet port of the CSU22. The microchannel was placed on the stage of an inverted microscope and the flow rate $(\mathrm{Q})$ of the working fluids was kept constant at $0.15 \mu \mathrm{l} / \mathrm{min}$ using a syringe pump (KD Scientific, Holliston, MA, USA), corresponding to a Reynolds number of 0.025 .

The laser beam was illuminated from below the microscope stage through a $20 \times$ objective dry lens with a numerical aperture (NA) equal to 0.75 . Satisfactory illumination was achieved by seeding $1 \mu \mathrm{m}$ diameter fluorescent particles that absorb green light (absorbance peak $542 \mathrm{~nm}$ ) and emit red light 
(emission peak $612 \mathrm{~nm}$ ). The light emitted from the fluorescent flowing particles passes through a colour filter into the CSU22 scanning unit, where it is reflected onto a high-speed camera using a dichromatic mirror to record the PIV images.

In order to obtain adequate quality images for processing with the PIV software (PivView; PivTec $\mathrm{GmbH}$, Göttingen, Germany), we captured images with a resolution of $640 \times 480$ pixels and 12-bit greyscale, at a rate of $200 \mathrm{frames} / \mathrm{s}$, with an exposure time of $4995 \mu \mathrm{s}$, and a time interval $(\Delta \mathrm{t})$ of $5 \mathrm{~ms}$ and $10 \mathrm{~ms}$ between two images. All the PIV measurements were performed for a period of approximately $0.5 \mathrm{~s}$ in order to obtain both instantaneous and ensemble averaged velocities. After recording the images, they were digitised and transferred to a computer for evaluation using Phantom camera control software (PH607). The PIV images of the flowing particles were processed and the flow velocity was determined using PivView version 2.3 (Rafael et al., 1998). The images were evaluated using a cross-correlation method in which the time between two images was set to $5 \mathrm{~ms}$ and $10 \mathrm{~ms}$ for all working fluids. Using a multiple-pass interrogation algorithm with a $24 \times 16$ pixel interrogation window (50\% overlap), which corresponds to a spatial resolution of $28.24 \times 18.83 \mu \mathrm{m}$, it was possible to obtain the corresponding instantaneous and ensemble velocity fields. A full description and evaluation of the confocal micro-PIV system used in this study can be found in Lima et al. (2006).

\section{Results and discussion}

\subsection{Ensemble velocity profiles}

Most previous studies have determined the velocity profiles of flowing blood by measuring the timeaveraged velocity field. Figure $2 \mathrm{a}$ and $1 \mathrm{~b}$ show the averaged velocity of $100(\Delta \mathrm{t}=5 \mathrm{~ms})$ and $50(\Delta \mathrm{t}=$ $10 \mathrm{~ms})$ ensemble PIV images, respectively. These images were recorded at the same time period of approximately $0.5 \mathrm{~s}$. Figure 2 also compares the PIV measurements with an analytical solution for steady flow through a long, straight, rigid square microchannel (see Lima et al., 2006 for more details).

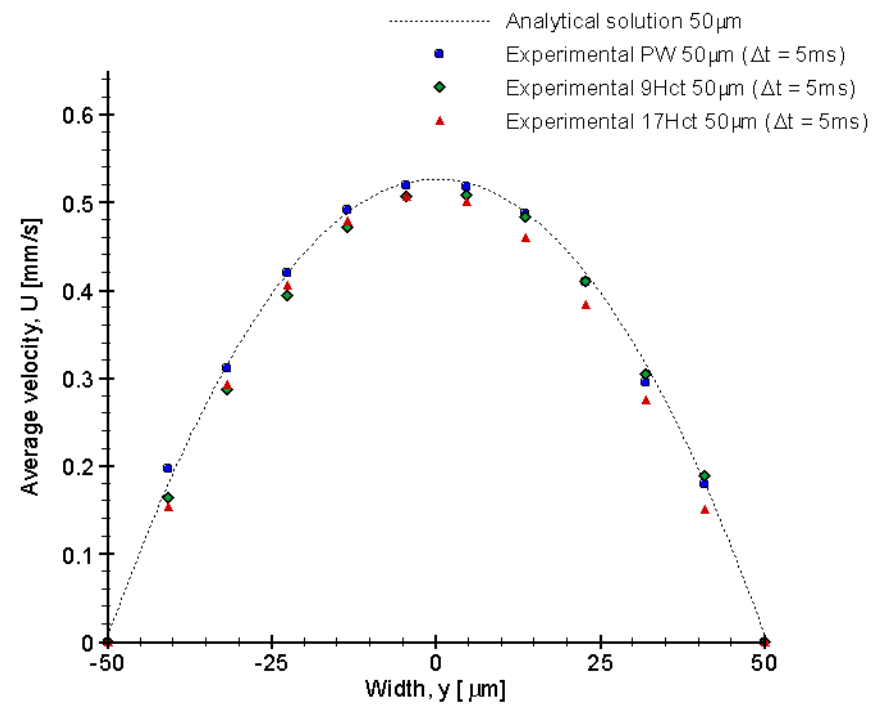




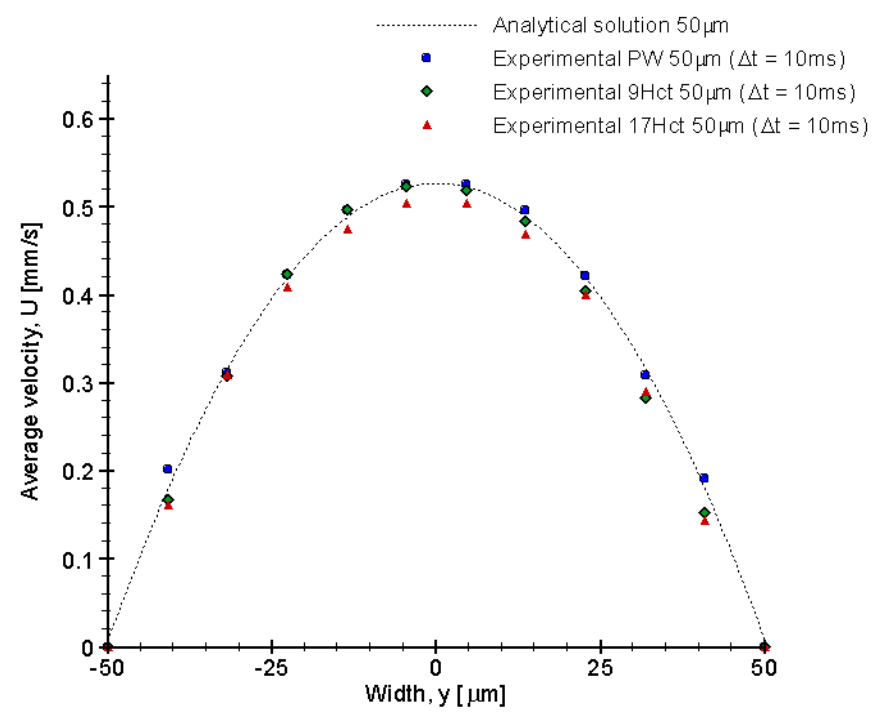

b)

Figure 2 Averaged ensemble velocity profiles for six different series of confocal micro-PIV measurements in the central plane $(50 \mu \mathrm{m})$ of (a) pure water (PW) and in vitro blood with haematocrit $9 \%$ (9Hct) and $17 \%$ (17Hct) for $\Delta \mathrm{t}=5 \mathrm{~ms}$; (b) pure water $(\mathrm{PW})$ and in vitro blood $(9 \mathrm{Hct}$ and $17 \mathrm{Hct})$ for $\Delta \mathrm{t}=10 \mathrm{~ms}$.

Comparing the ensemble velocity profiles of all fluids (see Figure 2), we observed small deviations (< $5 \%$ ) for both $\Delta \mathrm{t}=5 \mathrm{~ms}$ and $\Delta \mathrm{t}=10 \mathrm{~ms}$, especially in the central region of the velocity profile. Using the t-test analysis we found no significant difference between the working fluids and the analytical solution at $98 \%$ confidence interval. Hence, these results imply that the ensemble-averaged velocity profiles of in vitro blood with haematocrits up to $17 \%$ flowing within a $100 \mu \mathrm{m}$ square microchannel do not change significantly from a parabolic shape. These results agree with Baker et al. (1974) and Sugii et al. (2005). Furthermore, from the ensemble-averaged velocity profiles for both $\Delta \mathrm{t}=5 \mathrm{~ms}$ and $\Delta \mathrm{t}=10 \mathrm{~ms}$ the average deviation was estimated to be $2 \%$ for PW and $17 \% \mathrm{Hct}$ and $5 \%$ for $9 \% \mathrm{Hct}$. On comparing the results from both $\Delta \mathrm{t}$ we found no significant difference between $\Delta \mathrm{t}=5 \mathrm{~ms}$ and $\Delta \mathrm{t}=$ $10 \mathrm{~ms}$ at $98 \%$ confidence interval. These results suggest that for both cases it is possible to obtain reliable ensemble-averaged velocity profiles for all the working fluids used in this study.

\subsection{Instantaneous velocity profiles and root mean square (RMS)}

A remarkable advantage of the PIV measuring technique over conventional methods, such as doubleslit photometry or laser-Doppler anemometry, is its ability to obtain detailed information on the fluid flow behaviour from the evaluation of the instantaneous velocity fields. Although these velocity fields are extremely important for flows with high Re in which turbulent flow fields are likely to occur (Meinhart et al., 2000b; Bates et al., 2001; Heise et al., 2004), we believe that instantaneous velocities are also crucial for evaluating several phenomena in steady flows, especially for complex fluids containing suspended blood cells in plasma. In this way, Figures 3-4 show the first time series of the instantaneous velocity profiles of pure water and in vitro blood with two different haematocrits at $\mathrm{Re}$ 0.025 for $\Delta \mathrm{t}=10 \mathrm{~ms}$. 

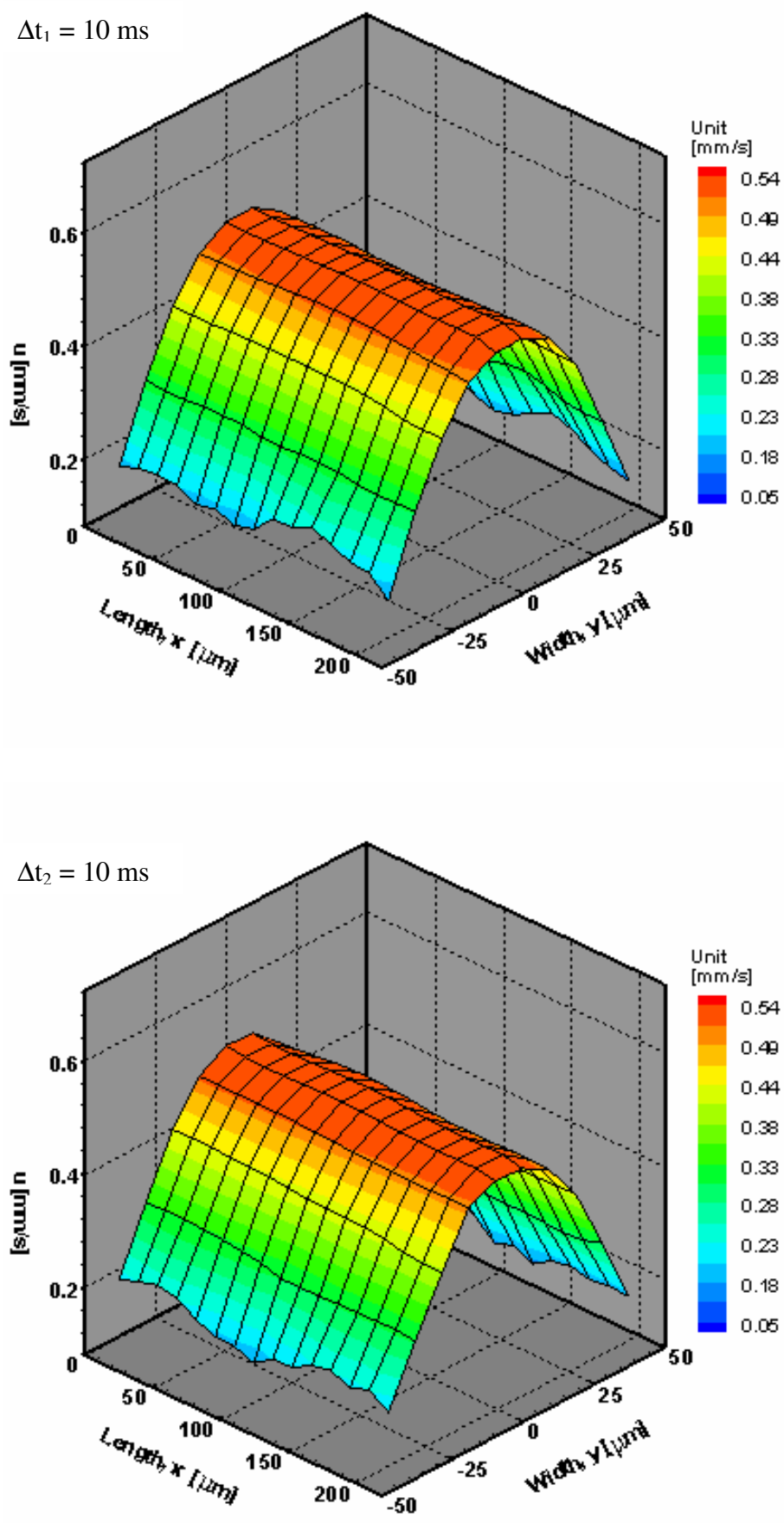

Figure 3 Two examples showing series of instantaneous velocity profiles of pure water $(\mathrm{PW})$ in the central plane $(50 \mu \mathrm{m})$ of the microchannel for $\Delta \mathrm{t}=10 \mathrm{~ms}$. Note that, $\mathrm{x}$, y represent respectively measured length and full width of the microchannel whereas $u$ represents the axial velocity of the working fluid along the central plane of the microchannel. 

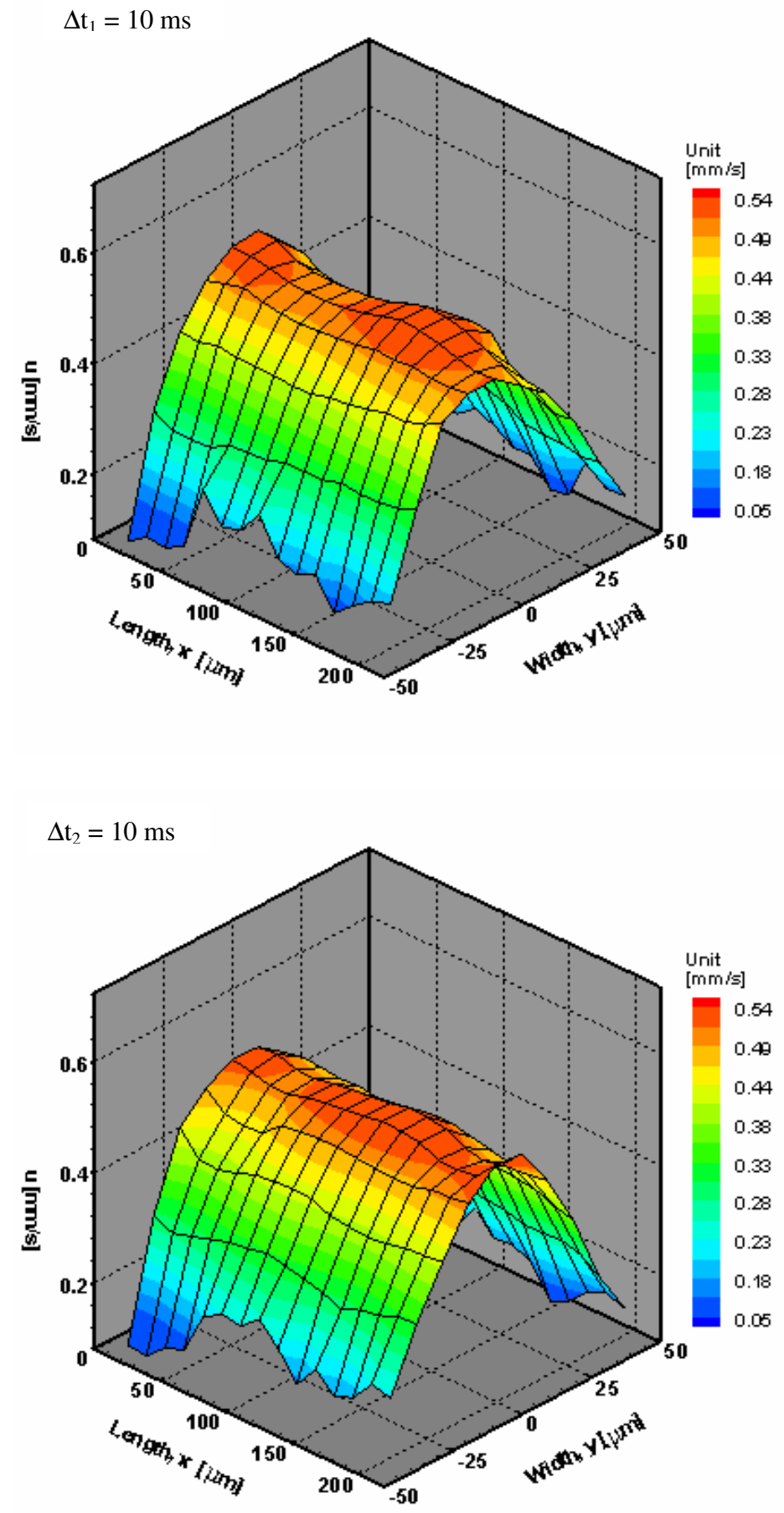

Figure 4 Two examples showing series of instantaneous velocity profiles of in vitro blood with a 9\% (9Hct) haematocrit in the central plane of the microchannel for $\Delta \mathrm{t}=10 \mathrm{~ms}$. 

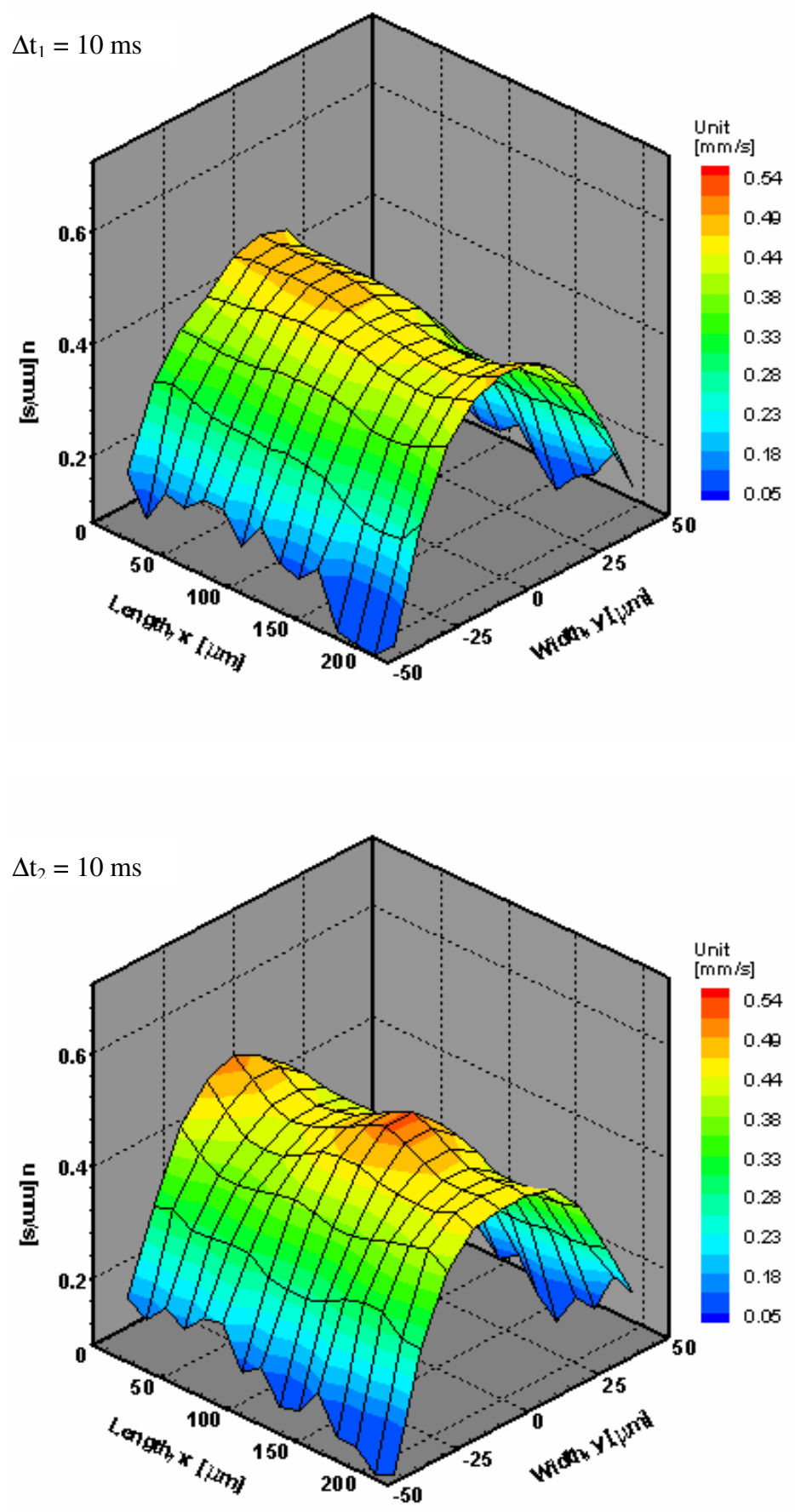

Figure 5 Two examples showing series of instantaneous velocity profiles of in vitro blood with a $17 \%$ (17Hct) haematocrit in the central plane of the microchannel for $\Delta \mathrm{t}=10 \mathrm{~ms}$.

In the conventional micro-PIV, most of the noise in the instantaneous velocity measurements is mainly due to the out-of-focus particle images, Brownian motion and low particle image density (Santiago et al., 1998; Nguyen and Wereley, 2002). The standard method to improve the accuracy of the conventional micro-PIV measurements is by ensembling series of instantaneous velocities. However, 
very recently it was shown that confocal micro-PIV, due to its optical sectioning effect provided by the spinning disk, can improve significantly the background noise even when using high particle concentration, i. e., $0.1 \%$ by volume (Park et al., 2004, 2006; Lima et al., 2006). Furthermore by using trace particles with $1 \mu \mathrm{m}$ diameter it is possible to minimize some possible Brownian motion effect (Santiago et al., 1998). As a result, we believe that confocal micro-PIV can provide reliable instantaneous velocity fields, especially for homogenous fluids and in vitro blood with low heamatocrit $(\mathrm{Hct} \leq 9 \%)$.

On comparing the instantaneous velocity fields in the middle plane of pure water and in vitro blood (see Figures 3-5), it is possible to observe that the instantaneous velocities from pure water have a nearly constant parabolic profile, whereas the instantaneous velocities from both in vitro blood show some irregularities on the velocity profiles. However, these instantaneous velocity profiles are only qualitative observations which needed to be quantified in order to understand the possible causes of the fluctuations encountered on the in vitro blood instantaneous velocity profiles (see Figures 4 and 5).

In an attempt to elucidate the possible causes of these findings we have calculated the Root Mean Square (RMS) which can be determined from the ensemble averaging of the instantaneous velocity measurements. To calculate the RMS we first need to determine the standard deviation of both $\mathrm{u}$ and $\mathrm{v}$ components of the velocity at each grid point for a series of $\mathrm{N}$ frames by using the following equations:

mean velocity components :

$$
\bar{u}=\frac{1}{N} \sum_{i=1}^{N} u_{i}, \quad \text { (1) } \quad \bar{v}=\frac{1}{N} \sum_{i=1}^{N} v_{i},
$$

standard deviation of $\mathrm{u}$ and $\mathrm{v}$ components :

$$
\sigma_{u}=\sqrt{\frac{\sum_{i=1}^{N}\left(u_{i}-\bar{u}\right)^{2}}{N-1}},
$$

$$
\sigma_{v}=\sqrt{\frac{\sum_{i=1}^{N}\left(v_{i}-\bar{v}\right)^{2}}{N-1}} .
$$

After calculating both $\sigma_{\mathrm{u}}$ and $\sigma_{\mathrm{v}}$, RMS can be estimated by

$$
R M S=\sqrt{\sigma_{u}^{2}+\sigma_{v}^{2}} .
$$

In Figure 6 the averaged RMS values for the three working fluids at two different $\Delta \mathrm{t}$ are plotted as a function of the microchannel width. 


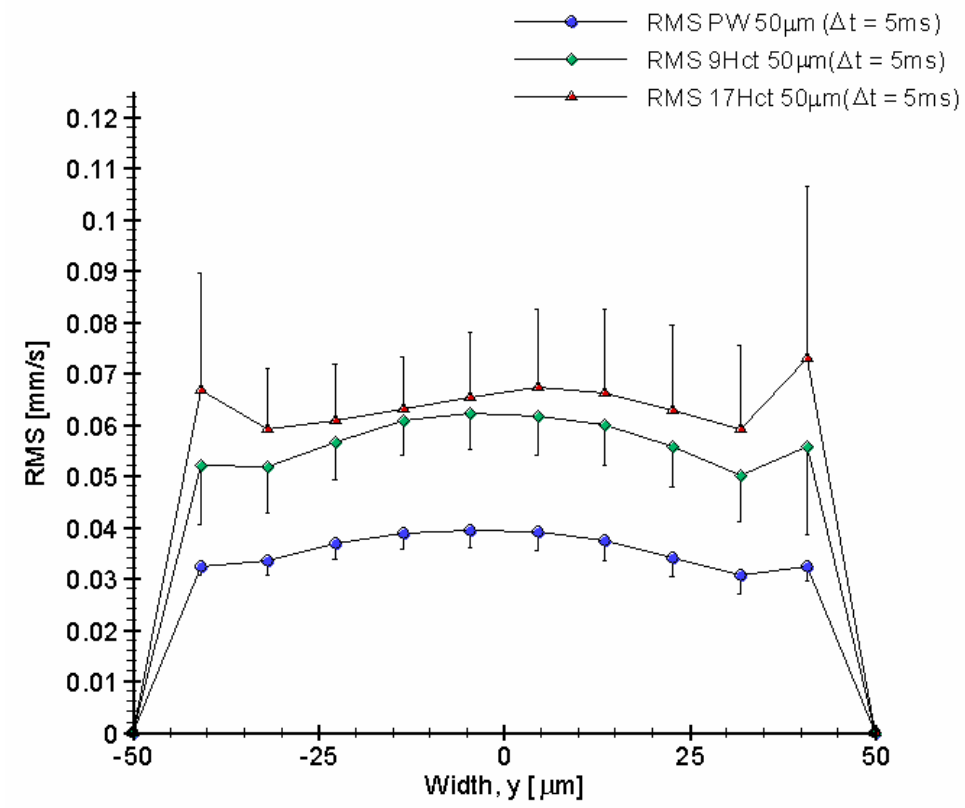

a)

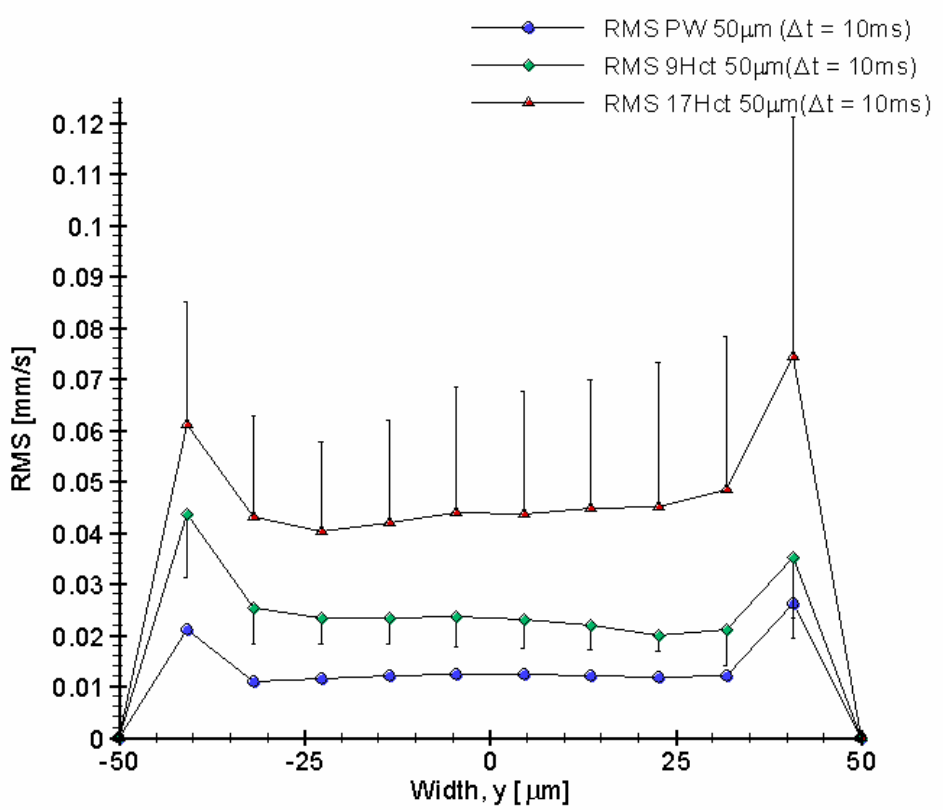

b)

Figure 6 Comparison of the RMS values for pure water (PW) and in vitro blood with haematocrits of $9 \%$ (9Hct) and $17 \%$ (17Hct) with (a) $\Delta \mathrm{t}=5 \mathrm{~ms}$, (b) $\Delta \mathrm{t}=10 \mathrm{~ms}$.

On comparing the RMS values with different $\Delta \mathrm{t}$ it is possible to observe that by increasing the time interval between two images the RMS decreases of about 50\% for PW and 9\% Hct and $25 \%$ for $17 \%$ Hct. Therefore, using $\Delta \mathrm{t}=10 \mathrm{~ms}$ the accuracy of the instantaneous velocity measurements are improved implying that the correspondent RMS values may represent a more reliable qualitative information about the time-dependent behaviour of the flow. Moreover, on comparing the RMS values from all the working fluids we have found that there is a significant difference between PW, 9\% Hct and $17 \%$ Hct at $90 \%$ confidence interval, except for some values near the wall (see Figure 6). Despite the inclusion of some bias errors in the RMS values, these results show clearly that the RMS increases 
with the haematocrit. We believe that the RMS values are strongly related to the fluctuations encountered in the instantaneous velocity profiles shown in Figures 4-5. The reasons for these small fluctuations are still not completely clear, however from the visualization of the RBCs motion through the microchannel, both rotational and tumbling motion and also the interaction between the neighbouring RBCs seem to be important factors to take into account. An ongoing study to clarify the effect of the RBCs on the plasma flow is currently under way.

It should be noted that for heamatocrits of about $17 \%$ we have observed some small random "plasma pockets" without particles in the PIV images which have created velocity vectors with small magnitudes due mainly to the light scattered and absorbed from the high concentration of RBCs within the plasma flow. Therefore, the RMS values for Hct $=17 \%$ contains quantitative information about not only the effect of the RBCs on the plasma flow but also some bias errors. One way to overcome this limitation is by increasing the particles concentration within the plasma or by using a rectangular microchannel with low aspect ratio which creates an uniform distribution of RBCs.

\subsection{Measurement noise and accuracy of the confocal micro-PIV system}

Past studies have already evaluated the accuracy of the confocal micro-PIV system to measure both homogenous and non-homogenous fluids (Park et al. 2004, 2006, Lima et al. 2006). However neither of them has investigated the influence of the light scattered and absorbed from the RBCs on the instantaneous velocity measurements. An approach to evaluate this effect is by calculating the Signalto-Noise Ratio (SNR) for the working fluids used in this study. The measured SNR was defined as the highest peak image intensity divided by average background intensity in the predefined interrogation window. Figure 7 shows representative correlation planes for the working fluids using both confocal and conventional micro-PIV whereas Figure 8 shows the correspondent averaged SNR. Note that the conventional micro-PIV measurements were obtained for pure water under identical specified flow conditions by using an epifluorescent microscope equipped with a mercury lamp and colour filters.

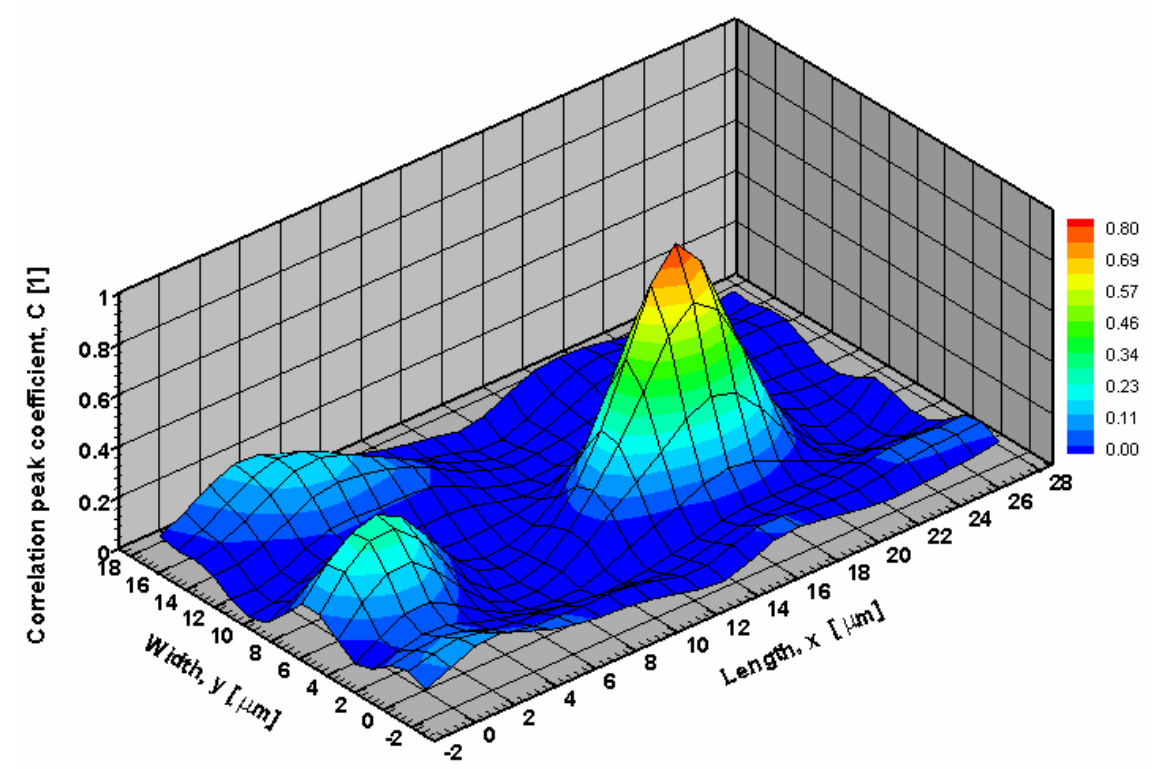

a) 


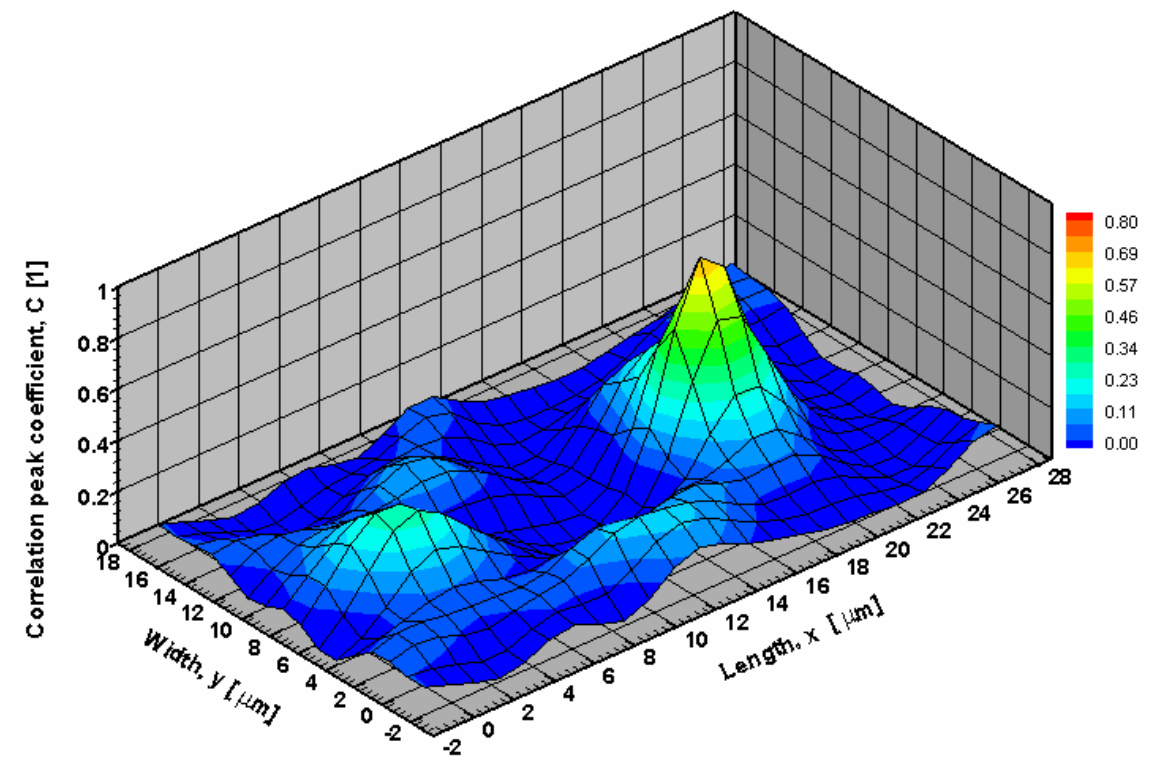

b)

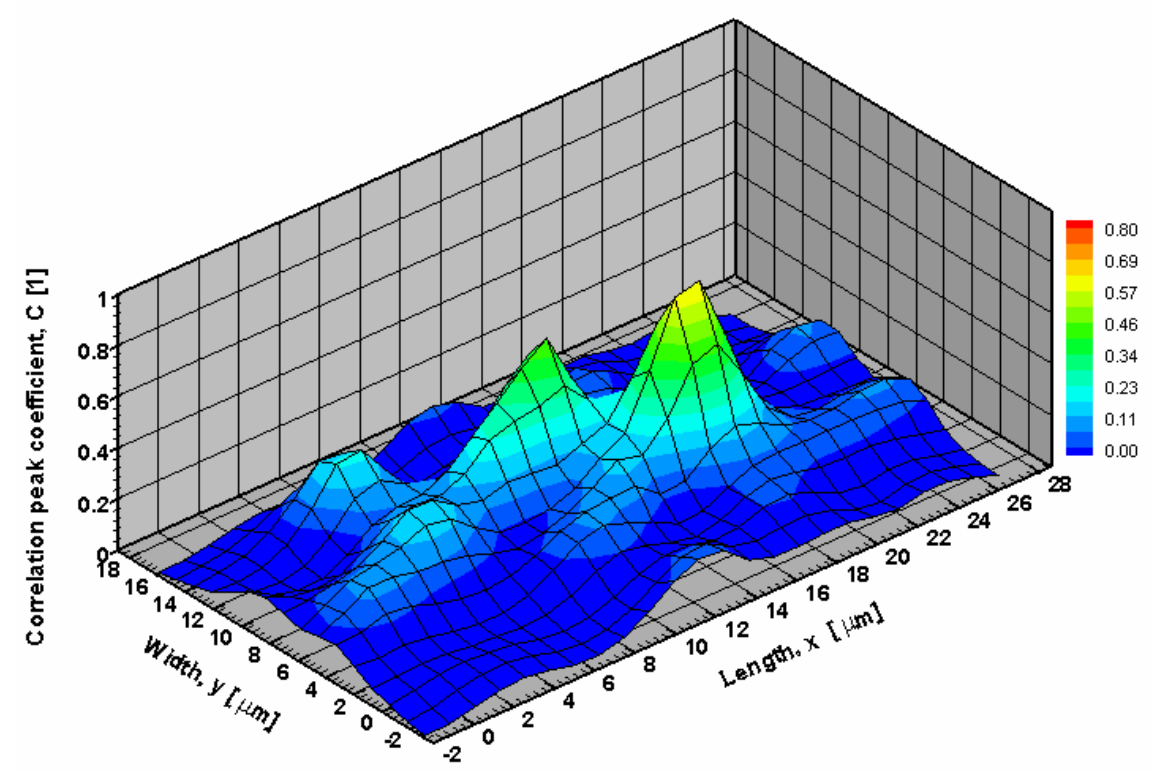

c) 


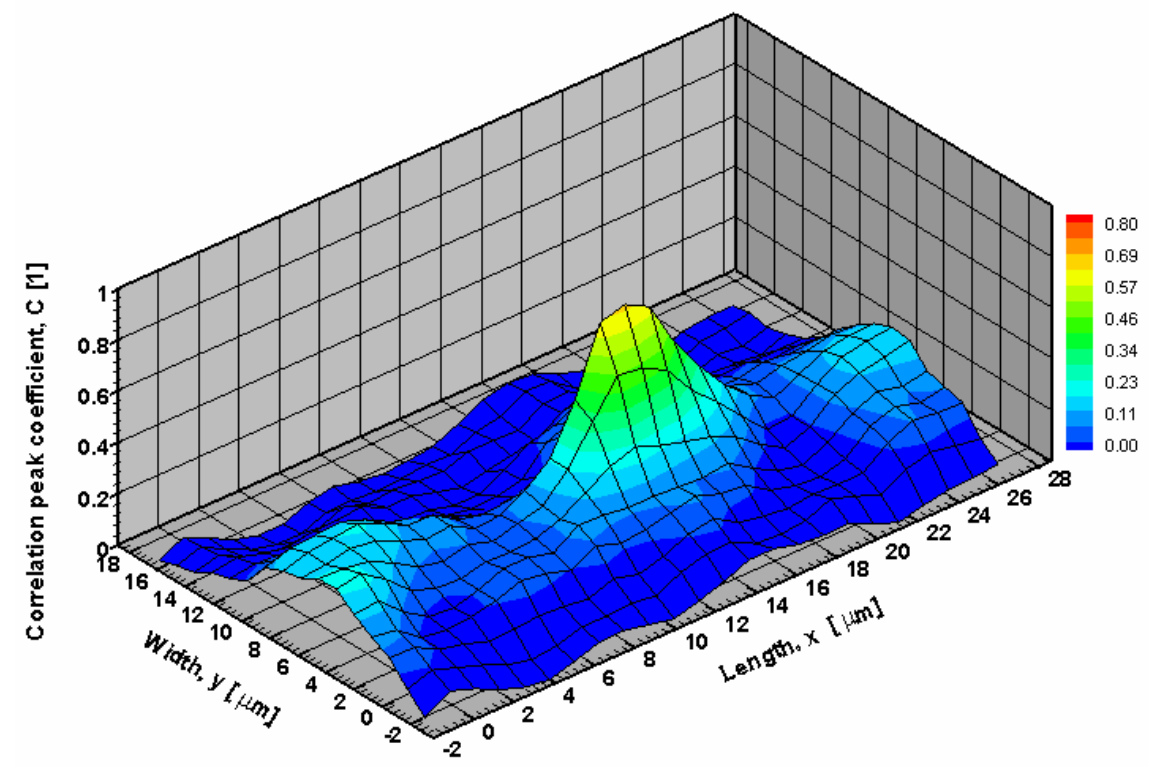

d)

Figure 7 Representative correlation planes for (a) pure water (PW), (b) in vitro blood with $\mathrm{Hct}=9 \%$, (c) in vitro blood with Hct=17\% using a confocal system and (d) PW using a conventional micro-PIV technique. The $\mathrm{x}$ and y axis correspond to the interrogation window $(28.24 \times 18.83 \mu \mathrm{m})$ used to obtain both instantaneous and ensemble velocity fields.

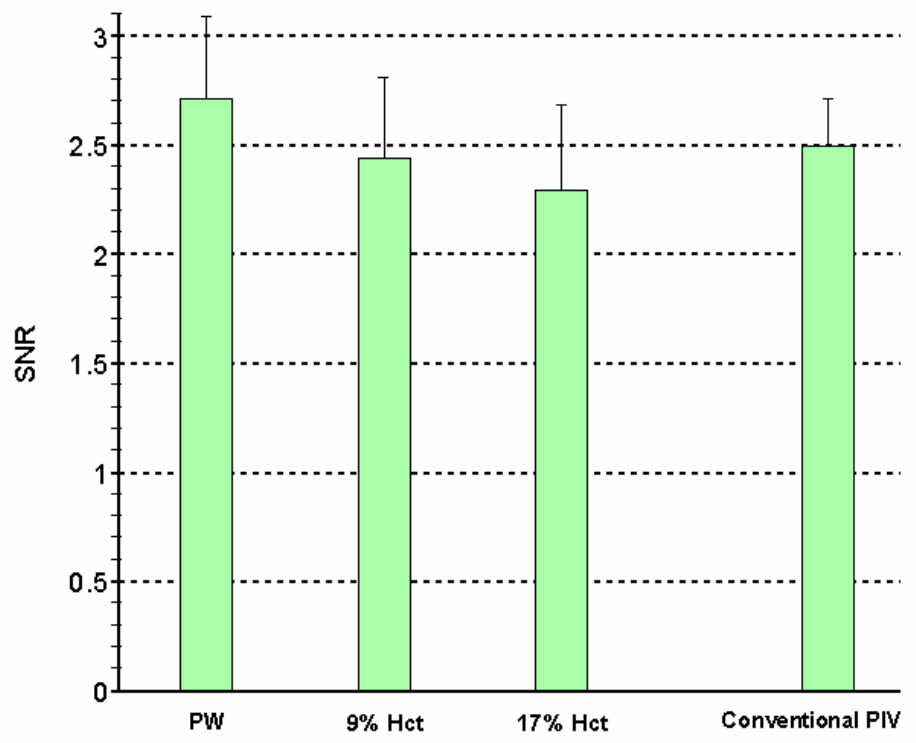

Figure 8 Comparison of the Signal-to-Noise Ratio (SNR) for PW, in vitro blood with haematocrits of 9\% (9Hct) and $17 \%$ (17Hct) using a confocal system and PW using a conventional micro-PIV technique. The measured values of the SNR are expressed as the means \pm standard deviation.

From Figure 7 it is possible to observe that for the case of the confocal system the correlation coefficient peak $(\mathrm{C})$ for pure water is the highest with a value of about 0.8. By increasing the Hct the 
main peak tends to become weak $(0.7$ for $9 \% \mathrm{Hct}$ and 0.6 for $17 \% \mathrm{Hct}$ ) due to the reduction of the number of particle pairs within the interrogation window. Nevertheless, the main peak for in vitro blood is still bigger than the noisy peaks. Furthermore, it is also possible to observe a decrease of the SNR with the increase of the Hct (see Figure 8).

Several researchers in microfluidics have performed successful measurements on both pure water (Santiago et al. 1998) and in vitro blood (Sugii et al. 2005) by using a conventional micro-PIV. Accordingly, we have decided to compare the SNR of pure water for both confocal and conventional micro-PIV system. The images obtained from the confocal system showed a much clearer image definition of the particles in comparison with the conventional system. We believe that the improvement on the image quality provided by the confocal system reflects the highest SNR shown in Figure 8. Nevertheless, the particle images with SNR values in the order of 2.5 seem to have adequate quality to obtain reliable velocity fields by using conventional correlation interrogation techniques. Thus, in vitro blood with $9 \%$ Hct seems to have enough SNR to generate reliable instantaneous velocity fields. For the case of $\mathrm{Hct}=17 \%$ it is still possible to obtain instantaneous velocities vectors however some outliers can be produced due mainly to the light scattered and absorbed from the high concentration of RBCs. In this way the instantaneous velocities fields for in vitro blood with $17 \% \mathrm{Hct}$ blood still require further investigations. One way to overcome the limitation of having lower particle concentration is by using the particle tracking velocimetry method (Ravnic et al. 2006).

\section{Conclusions}

In this study, we determined both ensemble and instantaneous velocity profiles for in vitro blood (haematocrit up to 17\%) flowing through a $100 \mu \mathrm{m}$ square microchannel. Although the ensemble velocity profiles were markedly parabolic, some fluctuations in the instantaneous velocity profiles were found to be closely related to the increase in the haematocrit. The present study shows clearly that the RMS values increase with the haematocrit implying that the presence of RBCs within the plasma flow strongly influences the measurements of the instantaneous velocity fields. The possible reasons for the RMS increase are the motion and interaction of RBCs and the light scattered and absorbed from the RBCs. This latter cause seems to be more predominant at $\mathrm{Hct}=17 \%$. As a result, for $17 \%$ Hct improvements on the Signal-to-Noise Ratio are required to further enhance the measurement performance of the instantaneous velocities.

\section{Acknowledgements}

This study was supported in part by the following grants: International Doctoral Program in Engineering from the Ministry of Education, Culture, Sports, Science and Technology of Japan (MEXT), "Revolutionary Simulation Software (RSS21)" next-generation IT program of MEXT; Grants-in-Aid for Scientific Research from MEXT and JSPS Scientific Research in Priority Areas (768) "Biomechanics at Micro- and Nanoscale Levels," Scientific Research (A) No.16200031 "Mechanism of the formation, destruction, and movement of thrombi responsible for ischemia of vital organs". The authors would like to thank Dr. Takuji Ishikawa for his valuable suggestions and discussion.

\section{References}

Baker, M., Wayland, H., 1974. On-line volume flow rate and velocity profile measurement for blood in microvessels. Microvascular Research 7, 131-143.

Bates, C., O`Doherty, D., Williams, D., 2001. Flow instabilities in a graft anstomosis: a study of instantaneous velocity fields. Proceedings of the Institution of Mechanical Engineers Part H 215, 579-587.

Born, G., Melling, A., Whitelaw, J., 1978. Laser Doppler microscope for blood velocity measurement. Biorheology 15, 163-172.

Bugliarello, G., Hayden, J., 1963. Detailed characteristics of the flow of blood in vitro. Transactions of The Society Rheology 7, 209-230.

Cochrane, T., Earnshaw, J., Love, A., 1981. Laser Doppler measurement of blood velocity in microvessels. Medical and Biological Engineering and Computing 19, 589-596.

Einav, S., Berman, R., Fuhro, P., DiGiovanni, P., Fine, S., Fridman, J., 1975. Measurement of velocity profiles of red blood cells in the microcirculation by laser Doppler anemometry (LDA). Biorheology 12, 207-210.

Gaehtgens, P., Meiselman, H., Wayland, H., 1970. Velocity profiles of human blood at normal and reduced hematocrit in glass tubes up to $130 \mu$ diameter. Microvascular Research 2, 13-23. 
Golster, H., Linden, M., Bertuglia, S., Colantuoni, A., Nilsson, G., Sjoberg, F., 1999. Red blood cell velocity and volumetric flow assessment by enhanced high-resolution laser Doppler imaging in separate vessels of hamster cheek pouch microcirculation. Microvascular research 58, 62-73.

Heise, M., Schmidt, S., Kruger, U., Ruckert, R., Rosler, S., Neuhaus, P., Settmacher, U., 2004. Flow pattern and shear stress distribution of distal end-to-side anastomoses. A comparison of instantaneous velocity fields obtained by particle image velocimetry. Journal of Biomechanics 37, 1043-1051.

Jeong, J. H., Sugii, Y., Minamiyama, M., Takeuchi, H., Okamoto, K., 2006. Interaction between liposomes and $\mathrm{RBC}$ in microvessels in vivo. Microvascular Research (in press).

Kinoshita, H., Oshima, M., Kaneda, S., Fujii, T., 2005. Confocal micro-PIV measurement of internal flow in a moving droplet. In: Proceedings of the $9^{\text {th }}$ International Conference on Miniaturized Systems for Chemistry and Life Sciences. Boston, MA, USA.

Lima, R., Wada, S., Tsubota, K., Yamaguchi, T., 2006a. Confocal micro-PIV measurements of three dimensional profiles of cell suspension flow in a square microchannel. Measurement Science and Technology 17, 797808.

Lima, R., Wada, S.,Tanaka, S., Takeda, M., Tsubota, K., Ishikawa T., Yamaguchi, T., 2006b. Velocity measurements of blood flow in a rectangular PDMS microchannel assessed by confocal micro-PIV system. In: Proceedings of the World Congress on Medical Physics and Biomedical Engineering. Seoul, pp. 278281.

Meinhart, C., Wereley, S., Santiago, J., 2000. A PIV algorithm for estimating time-averaged velocity fields. Journal of Fluids Engineering 122, 285-289.

Nakano, A., Sugii, Y., Minamiyama, M., Niimi, H., 2003. Measurement of red cell velocity in microvessels using particle image velocimetry (PIV). Clinical Hemorheology and Microcirculation 29, 445-455.

Nakano, A., Sugii, Y., Minamiyama, M., Seki, J., Niimi, H., 2005. Velocity profiles of pulsatile blood flow in arterioles with bifurcation and confluence in rat mesnetery measured by particle image velocimetry. JSME International Journal C 48 (4), 444-452.

Nguyen, N., Wereley, S., 2002. Fundamentals and applications of microfluidics. Norwood, MA: Artech House Inc., USA.

Park, J., Choi, C., Kihm, K., 2004. Optically sliced micro-PIV using confocal laser scanning microscopy (CLSM). Experiments in Fluids 37, 105-119.

Park, J., Kihm, K., 2006. Use of confocal laser scanning microscopy (CLSM) for depthwise resolved microscaleparticle image velocimetry ( $\mu$-PIV). Optics and Lasers in Engineering 44, 208-223.

Parthasarathi, A., Japee, S., Pittman, R., 1999. Determination of red blood cell velocity by video shuttering and image analysis. Annals of Biomedical Engineering 27, 313-325.

Raffel, M., Willert, C., Kompenhans, J., 1998. Particle image velocimetry: a practical guide, Springer-Verlag, Germany.

Ravnic, D., Zhang, Y., Tsuda, A., Pratt, J., Huss, H., Mentzer, S., 2006. Multi-image particle tracking velocimetry of the microcirculation using fluorescent nanoparticles. Microvascular Research 72, 27-33.

Santiago, J., Wereley, S., Meinhart, C., Beebe, D.,Adrian, R., 1998. A particle image velocimetry system for microfluidics. Experiments in Fluids 25, 316-319.

Sugii, Y., Nishio, S., Okamoto, K., 2002. In vivo PIV measurement of red blood cell velocity field in microvessels considering mesentery motion. Physiological Measurement 23, 403-416.

Sugii, Y., Okuda, R., Okamoto, K., Madarame, H., 2005. Velocity measurement of both red blood cells and plasma of in vitro blood flow using high-speed micro PIV technique. Measurement Science and Technology 16, 1126-1130.

Tangelder, G., Slaaf, D., Muijtjens, M., Arts, T., Egbrink, M., Reneman, R., 1986. Velocity profiles of blood platelets and red blood cells flowing in arteriols of rabbit mesentery. Circulation Research 59, 505-514.

Tanaani, T., Otsuki, S., Tomosada, N., Kosugi, Y., Shimizu, M., Ishida, H., 2002. High-speed 1-frame/ms scanning confocal microscope with a microlens and Nipkow disks. Applied Optics 41 (22), 4704-4708.

Uijttewaal, W., Nijhof, E., Heethaar, R., 1994. Lateral migration of blood cells and microspheres in twodimensional Poiseuille flow: a laser Doppler study. Journal of Biomechanics 27, 35-42. 